Research Article

\title{
The Research of Chronic Gastritis Diagnosis with Electronic Noses
}

\author{
Yifei Chen $\mathbb{D},{ }^{1}$ Rongfei Xia $\left(\mathbb{D},{ }^{2}\right.$ and Yongjian Feng $\mathbb{D}^{1}$ \\ ${ }^{1}$ School of Aerospace Engineering, Xiamen University, Xiangan South Road, Xiamen 361102, China \\ ${ }^{2}$ Chengyi University College, Jimei University, 199 Jimei Avenue, Xiamen 361021, China \\ Correspondence should be addressed to Yongjian Feng; yjfeng@xmu.edu.cn
}

Received 25 January 2021; Revised 9 August 2021; Accepted 16 November 2021; Published 2 December 2021

Academic Editor: Salvatore Pirozzi

Copyright (c) 2021 Yifei Chen et al. This is an open access article distributed under the Creative Commons Attribution License, which permits unrestricted use, distribution, and reproduction in any medium, provided the original work is properly cited.

\begin{abstract}
In order to solve the problem of existing diagnostic methods for chronic gastritis which are complex and traumatic, a novel noninvasive method for diagnosis of chronic gastric based on e-nose and deep convolutional neural network is proposed. Firstly, in order to collect samples, a respiratory gas sampling device was established and the response curve of respiratory gas is generated. Then, a deep convolutional neural network for the diagnosis of chronic gastritis is proposed to recognize and classify the respiratory gas response curve. The DCNN model attained good results with accuracy, sensitivity, and specificity of $85.00 \%, 90.00 \%$, and $80.00 \%$, respectively, for chronic gastric prediction. The proposed method provides a new way for the clinical auxiliary diagnoses of chronic gastric.
\end{abstract}

\section{Introduction}

Inspired by the principle of the biological olfactory, electronic nose is a new bionic detection equipment combined with modern sensor technology, electronics, and pattern recognition technology, with the ability to identify simple or complex odors [1-5]. Compared with the traditional gas detectors such as gas chromatography, mass spectrometry, and infrared spectroscopy, it has the advantages of small size, lightweight, low cost, and high accuracy $[6,7]$. The e-nose system generally consists of two parts: sensor array and pattern recognition algorithms. The sensor array responds to different odors and generates response curves. This means that the signals from the sensor array contain a large amount of information about the sample, which plays an important role in the performance of the e-nose. There are many kinds of gas sensors; commonly used are as follows: solid electrolyte gas sensor, semiconductor gas sensor, electrochemical gas sensor, and polymer gas sensor. As the performance of various sensors is very different, it is selected according to different detection objects. Metal oxide semiconductor sensor plays a key role in the field of gas sensor researches and applications with the advantages of high sensitivity and low cost $[8,9]$. Based on these advantages, the e-nose with metal oxide semiconductor sensor contains a large amount of information from complex gas sample.

E-nose has been widely used in the field of food industry, tobacco industry, medical diagnosis, and environmental monitoring because of its character such as noninvasive and easy to operate [10-14]. The application of e-nose in medical diagnosis especially has attracted more and more interest of the researchers. Liu et al. developed an e-nose system for lung cancer detection via breath with a sparse group feature selection approach and attained better results with 94.2\% accuracy, 97.8\% sensitivity, and $90.2 \%$ sensitivity [15]. Raspagliesi et al. tested the feasibility of e-nose as a diagnostic tool for ovarian cancer, the performance tested in the prediction gave $98 \%$ of sensitivity and $95 \%$ of specificity [16]. Li et al. fabricated an e-nose based on 10 gas sensors of 4 types and attained mean classification accuracy of 86.42\% using samples from 153 healthy participants and 115 lung cancer patients [17]. Hendrick et al. proposed a noninvasive method for tuberculosis-exhaled breath classification based on electronic nose [18]. There is a growing body of literature suggesting that e-nose could be considered a useful tool for the early detection of lung and head and 
neck cancers [19-22]. In these applications, the original data acquired from the sensor array cannot be used directly due to noise and drift, and the signal processed, filtered, and dimension reduction should be carried out first, which greatly affected the performance of e-nose, and different methods are selected according to difference objects, which lead to a large waste of time and energy.

In order to obtain more information from e-nose data, new effective pattern recognition algorithms and prediction models are urgently needed. A deep convolutional neural network (DCNN) shows powerful features in image classification and recognition applications [23]. The DCNN is an efficient recognition algorithm developed in recent years, which mainly includes feature extraction layers and mapping layers. The input samples can be used directly without any preprocessing. And the DCNN can learn independent characteristics, with the stronger discriminant power than traditional manual selected characteristics. It has been widely used in the fields of traffic, remote sensing, agriculture, and medicine and biology and mainly application in image classification and recognition [24-27]. Wei et al. proposed a new LeNet-5 convolutional neural network structure for electronic noses [28]. The final gas identification accuracy rate reached $98.67 \%$. Galanty et al. presented a tool that can facilitate the recognition of cladonia lichen species with deep convolutional neural network, and the trained model achieved $60.94 \%$ accuracy [29]. Their final experimental result has an accuracy of $95.2 \%$, which is higher than SVM and MLP. Wang et al. used a flow modulation method to exploit transient sensor information [30]. The proposed method uses fewer steps to achieve higher recognition accuracy and improve the recognition speed and to reduce the training and testing costs. Jiang et al. proposed an active learning ( $\mathrm{AL}$ ) algorithm based on improved query by committee (QBC) for RBFNN is to distinguish three indoor pollutant gases, and the classification accuracy has been improved when using unlabeled samples in an application process [31]. Yang et al. developed an ANN model for analysis of benzoic acid in cola-type-carbonated beverages [32]. The model generated accuracy rates of $90.0 \%$ in category identification for test samples. Peng et al. proposed a novel deep convolutional neural network with 38 layers for gas classification [33]. Experimental results show that the DCNN method is an effective technique for classifying electronic nose data. All these works show good prospective applications of deep learning methods in gas identification fields.

Chronic gastritis is a common digestive disease caused by different reasons. Clinical trials have found the patients with chronic gastritis with varying degrees of oral off-odors [34]. Zhenqin verified that e-nose can help distinguish benign and malignant gastric cancer, and the analysis of respiratory gas offers a new way to research methods in the early diagnosis of gastric cancer [35]. Xuejuan et al. analyzed the odor fingerprint map of respiratory gas from patients with chronic gastritis used e-nose and concluded that the odor fingerprint map of respiratory gas can be accurately identified and chronic gastritis differences of cold and hot syndromes can be discerned by e-nose [36]. At present, research on chronic gastritis using e-nose is still in the begin- ning and exploratory phase. The above researches are proved to be effective in the diagnosis of chronic gastric with respiratory gas response curve.

This paper studied the detection and classification of chronic gastritis, combining e-nose and DCNN, and proposed a new diagnosis method of chronic gastritis. The raw data of the e-nose is directly input in the form of gray value image without any operations such as data processing and feature extraction, which greatly simplifies the data processing of traditional methods. In this paper, a new model that can identify chronic gastritis has been established based on DCNN, which can effectively distinguish patients with chronic gastritis from healthy people, and further verified the feasibility of chronic gastritis detection used e-nose.

The main contributions of this paper are as follows: (1) a novel noninvasive method for diagnosis of chronic gastric based on breath analysis is proposed to solved the problem of vivo trauma detection. (2) It was proved that diagnosis of chronic gastritis through breath analysis is feasible, by comparing the responses of chronic gastritis patients and healthy volunteers and PCA analysis. (3) The experiments are designed and executed to prove the effectiveness of the improved DCNN model by comparing with the PCA+BP.

The paper is organized as follows. In Section 2, the respiratory gas acquisition system is presented and introduced in detail. Section 3 describe the improved DCNN model for detection of chronic gastritis. Section 4 discusses and evaluates the performance of the proposed model by experiment and analysis. Finally, the inclusion and future works are summarized in Section 5.

\section{The Respiratory Gas Acquisition System}

Medical electronic nose with $\mathrm{SnO}_{2}$ thin-film gas sensor arrays of EN0111103-A developed by professor $\mathrm{Wu}$ is used in this paper [37]. Figure 1 shows the overall structure of the sensor; the sensor consists of $4 \mathrm{SnO}_{2}$ thin-films, heater, and temperature measurement circuit. Figure 2 shows the structure of $\mathrm{SnO}_{2}$ film, the oxide semiconductor film materials mixed with different substances which provides gas selectivity. The respiratory gas acquisition system is shown in Figure 3. The system consists of two parts: the control computer and the sampling device. The control computer is composed of signal processing, power source, and the data sampling circuit. The sampling device is composed of a gas chamber, a injector port, and flow control equipment. The chamber size is $100 \times 50 \times 100 \mathrm{~mm}$, with 10 built-in thinfilm gas sensor arrays installed in the chamber. The operating temperature of the sensor is 200 degrees. The gas flow rate is controlled by a flow controller; when patients breathe into the sampling device, the respiratory gas attached to the semiconductor film and the resistance of the semiconductor film changes.

\section{The Proposed Model}

Inspired by the traditional Alexnet and application of other typical models [38-40], we proposed a deep convolutional neural network model for detection and identification of 


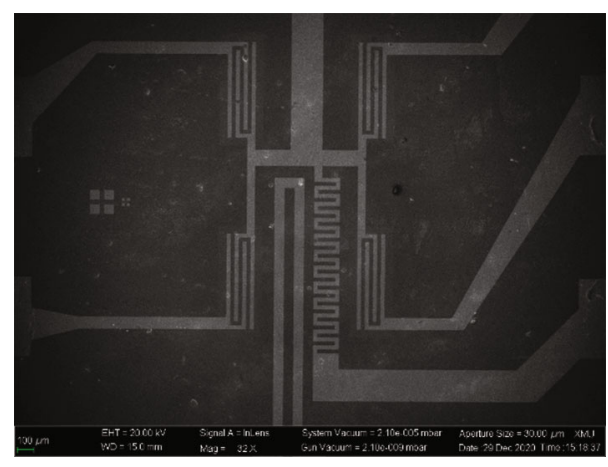

FIgURE 1: Overall structure of the sensor.

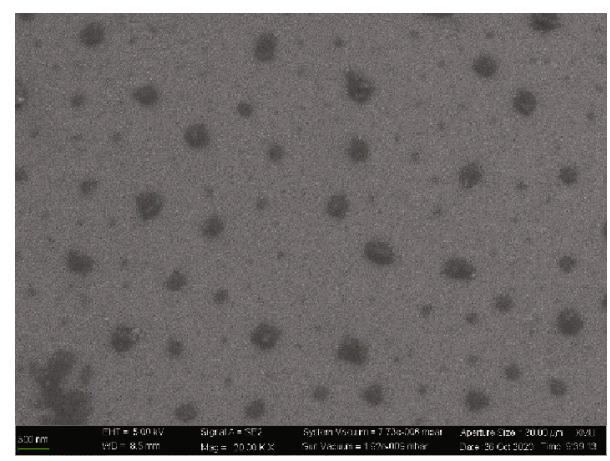

FIgURE 2: structure of $\mathrm{SnO}_{2}$ film.

chronic gastritis. The model structure is shown in Figure 4 . The characteristics of respiratory gas response curve is too less compared with the image, and the samples is few. That can lead to the model overfitting. The batch normalization $(\mathrm{BN})$ is added in the proposed model to solve this problem. Table 1 shows the detail of each layer of the proposed DCNN.

DCNN includes a convolutional layer, pooling layer, fully connected layer, and activation function. The DCNN proposed in this paper consists of three convolution layers and three full connection layers. BN is used after each convolution layer of the proposed DCNN to improve the efficiency of classification and learning of the networks by constraint the standardization of networks during training.

The size of input image is $56 \times 56 \times 3$ based on experience in this paper. Acquired response curves are convert to images as the input for the first convolutional layer, the size of convolution kernel is $11 \times 11$. And rectified linear unit (LeRU) is used as activation function after each convolution layer and as the input of pooling layer. The first $\mathrm{BN}$ is used between the first convolutional layer and LeRU activation function and as the input of the first pooling layer. What follows is the first pooling layer with max-pooling, the size of filter is $3 \times 3$, and it is used as the input of the second convolution layer. The second convolution layer has 256 convolution kernels with a size of $5 \times 5$, followed by the second $\mathrm{BN}$ and the second pooling layer with a filter size of $5 \times 5$. The third convolution layer has 384 convolution kernels with a size of $3 \times 3$, followed by the third BN and the third pooling layer with a filter size of $2 \times 2$. Next are the fully connected layers with 256 neurons followed by dropout to avoid overfitting. The last fully connected layer has 2 neurons as the number of output class, which is the output matrix we get.

After the output matrix is obtained, the soft-max activation function is used for multiclass classification. Then, calculate the loss between the predicted labels and the actual labels by sigmoid cross-entropy. The next step is to train the model and optimize the losses. A gradient descent method is a common method to solve optimization problems. Each gradient update will require all the database data, and the update speed is slow, so the model cannot be updated online. The improved momentum gradient method is used as the optimizer in this paper [41].

\section{Experiment and Analysis}

4.1. Experimental Data. This paper takes 600 chronic gastritis patients as the object for research. Among them, 285 were males and 315 were females, aged between 27 and 69. A total of 100 healthy volunteers were taken as the control group, including 50 males and 50 females.

In this experiment, 100 patients with chronic gastritis and 100 healthy volunteers were selected for chronic gastritis detection. And the dataset was randomly divided into two parts: $70 \%$ as the training set and $30 \%$ as the test set. Detected objects were required to fast for more than 12 hours to ensure the purity of breathing gas. The respiratory gas sampling device needs to run 1 hours in advance to reach the correct operating temperature of $200^{\circ} \mathrm{C}$. The ambient temperature is $15-35^{\circ} \mathrm{C}$, the relative humidity is less than $95 \%$, and airflow velocity is about $333 \mathrm{~m} \mathrm{~L} / \mathrm{min}$.

The response curves of the e-nose system correspond to the 10 gas sensors, respectively, as shown in Figure 5. The $x$ -axis represents the sampling time, while the sensor responses are reported on the $y$-axis.

From the response curves, each sensor has a different response to the same sample. Also, from the response curves, we can see that the 10 sensors went up from 0 to $170 \mathrm{sec}-$ onds, reached the peaks at 180 seconds, began to decline at 190 seconds, and became almost stable after 450 seconds. Overall, the responses of sensor array to chronic gastritis and healthy volunteers are different; it means that chronic gastritis is identified by sensor array would be feasible.

4.2. Experimental Results and Discussion. The proposed method has been implemented with Python Environment, a computer system with 32GB RAM, a NVIDIA GeForce GTX 1080 Ti graphics processor unit (GPU), and an Intel ${ }^{\circledR}$ Core $^{\mathrm{TM}}$ i7-8700K@3.70 GHz central processor unit (CPU). First, the response curves were classified and recognized, which is directly used as the input of the proposed DCNN. The response curves of 10 sensors are actually matrix of 10 $\times 500$. And we convert the matrix as gray value images and resize to $56 \times 56 \times 3$ as the input of the proposed DCNN. Figure 6 shows the response curves available as images.

The superiority of DCNN proposed in this paper was verified by experimental comparison with back propagation (BP) after principal component analysis (PCA) [42, 43]. The 


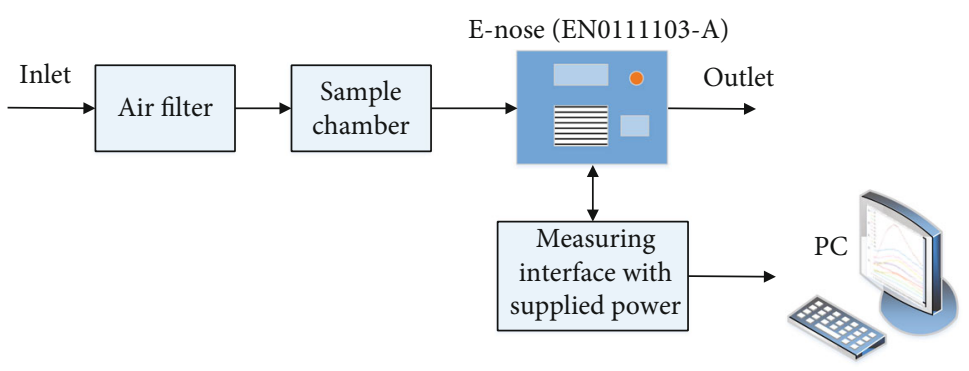

FIGURE 3: Respiratory gas acquisition system.

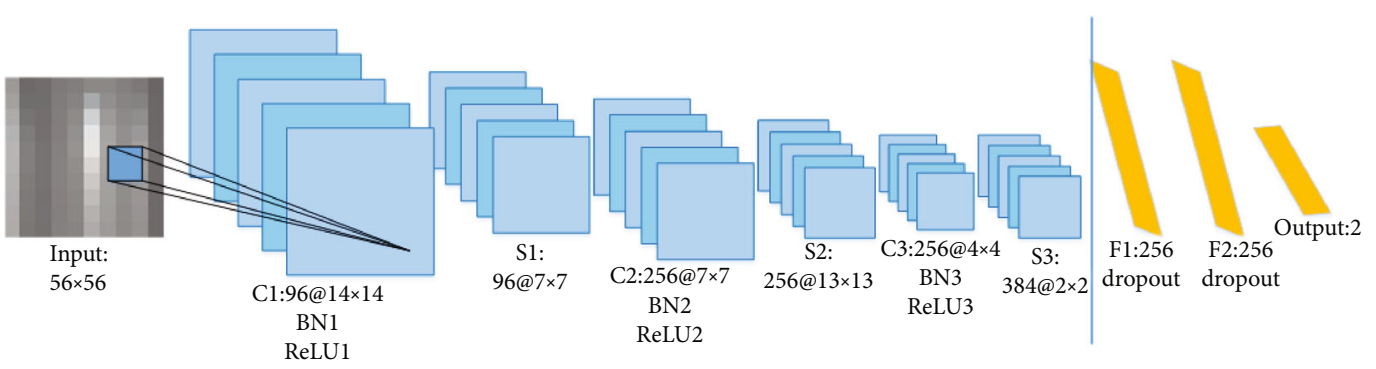

FIGURE 4: The architect of the proposed DCNN.

TABLE 1: Description of proposed twenty layer DCNN architecture.

\begin{tabular}{|c|c|c|c|c|c|}
\hline Layers & Layer name & Maps and neurons & Kernel & params & ops \\
\hline 0 & Image input & $3 @ 56 x 56$ & - & & \\
\hline 1 & Convolution & 96@14x14 & $11 \times 11$ & $12 \mathrm{~K}$ & $2.3 \mathrm{M}$ \\
\hline 2 & Batch normalization & Batch normalization & - & & \\
\hline 3 & $\mathrm{ReLu}$ & $\mathrm{ReLu}$ & - & & \\
\hline 4 & Max-pooling & 96@7x7 & $3 \times 3$ & & \\
\hline 5 & Convolution & 256@7x7 & $5 \times 5$ & $6 \mathrm{~K}$ & $0.3 \mathrm{M}$ \\
\hline 6 & Batch normalization & Batch normalization & - & & \\
\hline 7 & $\mathrm{ReLu}$ & $\mathrm{ReLu}$ & - & & \\
\hline 8 & Max-pooling & 256@4x4 & $3 \times 3$ & & \\
\hline 9 & Convolution & 384@4x4 & $3 \times 3$ & $4 \mathrm{~K}$ & $0.06 \mathrm{M}$ \\
\hline 12 & Batch normalization & Batch normalization & - & & \\
\hline 13 & $\mathrm{ReLu}$ & ReLu & - & & \\
\hline 14 & Max-pooling & $384 @ 2 x 2$ & $3 \times 3$ & & \\
\hline 16 & Fully connected & 256 & - & $393 \mathrm{~K}$ & $0.4 \mathrm{M}$ \\
\hline 17 & Dropout & Dropout & - & & \\
\hline 18 & Fully connected & 256 & - & $65 \mathrm{~K}$ & $0.07 \mathrm{M}$ \\
\hline 19 & Dropout & Dropout & - & & \\
\hline 20 & Classification output & 2 & - & 512 & 512 \\
\hline
\end{tabular}

response value at 180 seconds was selected and analyzed in the experiment. The contributing rate of each principal component is shown in Table 2. It can be seen from the table that the contribution rate of the first principal component (PC1) is $83.6 \%$ and the contribution rate of the second principal component (PC2) is $10.1 \%$. The first and second principal components were selected in this paper, and their additive contribution rate came up to $93.7 \%$. It contains almost all the information of the sensors. The correlation coefficients of 10 sensors are shown in Table 3.

Figure 7 is the analysis diagram of PCA for chronic gastritis. From the diagram, we can see that there are apparent differences between chronic gastritis and healthy volunteers, and they can be distinguished. The samples were forecasted using the BP network, input layer with 2 neurons, and output layer with 2 neurons corresponding 


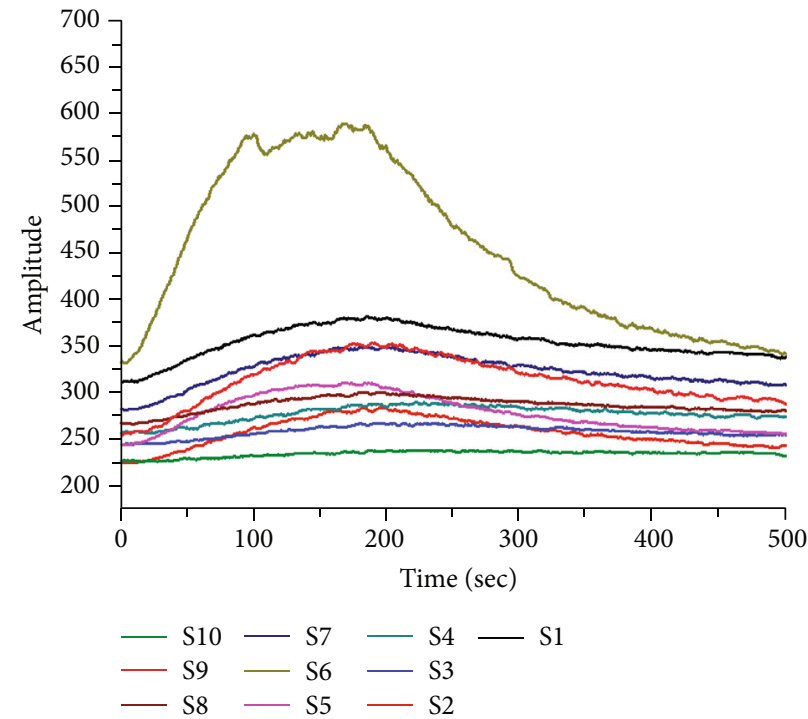

(a) Response curve of healthy volunteer

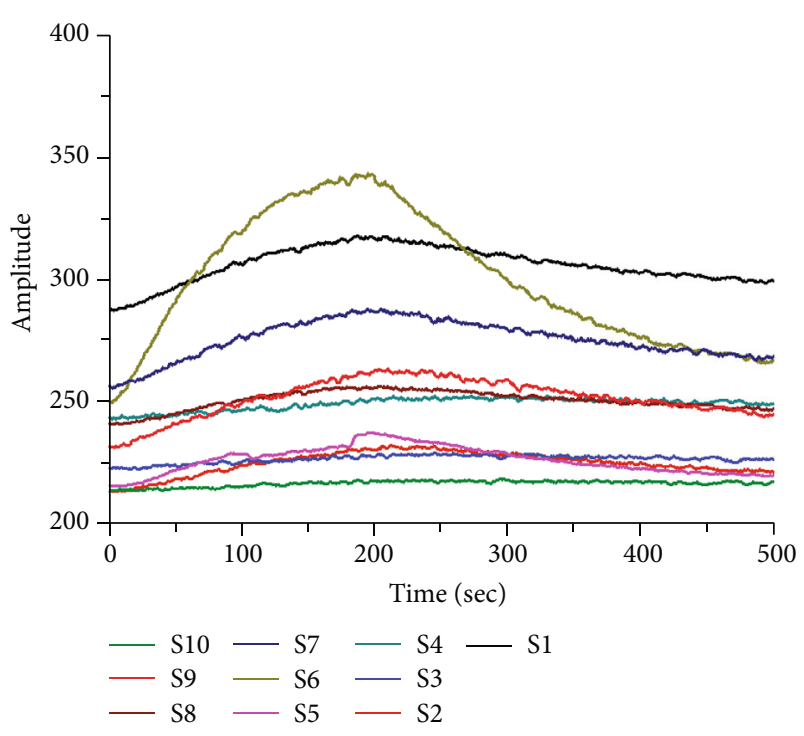

(b) Response curve of gastritis patient

FIgURE 5: Response curves of 10 sensors obtained from respiratory gas.

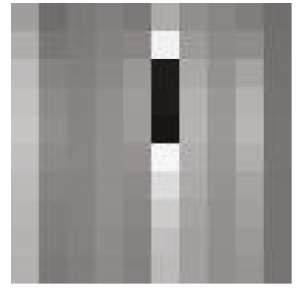

(a) Healthy volunteers

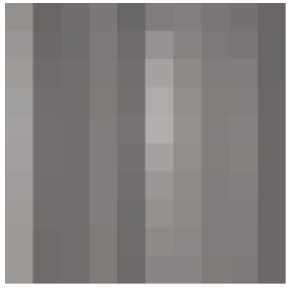

(b) Chronic gastritis
FIgURE 6: The gray value images of the response curve.

to the chronic gastritis and healthy volunteers; the number of iterations is 2000 .

To evaluate the performance of the method, the root mean squared error (RMSE) and correlation coefficients $\left(R^{2}\right)$ are adopted. RSME and $R^{2}$ are defined as Equations (1) and (2), respectively.

$$
\begin{aligned}
\mathrm{RMSE} & =\sqrt{\frac{1}{n} \sum_{i=1}^{n}\left(y_{i}-y \wedge_{i}\right)^{2}}, \\
R^{2} & =1-\frac{\sum_{i=1}^{n}\left(y_{i}-y \wedge_{i}\right)^{2}}{\sum_{i=1}^{n}\left(y_{i}-\bar{y}_{i}\right)^{2}},
\end{aligned}
$$

where $\hat{y}_{i}$ is the output value of the model, $y_{i}$ is actual value, $\bar{y}_{i}$ is the mean of actual values, and $n$ is the total number of samples.

The experimental results on the test data are shown in Figure 8. The experiment results showed that the proposed DCNN of $R^{2}=0.9787$ and RSME $=0.8513$ better than the $\mathrm{BP}$ of $R^{2}=0.97377$ and $\mathrm{RSME}=1.7649$. It shows that the proposed DCNN has higher precision of detection and identification for chronic gastritis, and the processing of the data is easier.
TABLE 2: Variance of each component by PCA.

\begin{tabular}{lccc}
\hline Number & $\begin{array}{c}\text { Characteristic } \\
\text { value }\end{array}$ & $\begin{array}{c}\text { Contribution } \\
(\%)\end{array}$ & $\begin{array}{c}\text { Total contribution } \\
(\%)\end{array}$ \\
\hline 1 & 9.32157 & 83.636 & 83.636 \\
2 & 1.12992 & 10.138 & 93.774 \\
3 & 0.35453 & 3.181 & 96.955 \\
4 & 0.16974 & 1.523 & 98.478 \\
5 & 0.10621 & 0.953 & 99.431 \\
6 & 0.05929 & 0.532 & 99.963 \\
7 & 0.00278 & 0.025 & 99.988 \\
8 & 0.00066 & 0.006 & 99.994 \\
9 & 0.00044 & 0.004 & 99.998 \\
10 & 0.00022 & 0.002 & 100 \\
\hline
\end{tabular}

In order to further verify the model's effectiveness and reliability, 60 samples (healthy: 30, chronic gastritis: 30 ) were selected for the experiment. For both approaches, the criteria such as accuracy, corresponding to the probability of doing a correct classification; sensitivity, corresponding to the probability to classify a subject as a case when this is true; and specificity, corresponding to the probability of classifying a subject as control when this is true have been utilized. The equations are given as follows:

$$
\begin{array}{r}
\text { Accuracy }=\frac{\mathrm{TP}+\mathrm{TN}}{n} \times 100 \%, \\
\text { Sensitivity }=\frac{\mathrm{TP}}{\mathrm{TP}+\mathrm{FN}} \times 100 \%, \\
\text { Specificity }=\frac{\mathrm{TN}}{\mathrm{FP}+\mathrm{TN}} \times 100 \%,
\end{array}
$$

where TP is the number of chronic gastritis correctly 
TABLE 3: The correlation of 10 sensors.

\begin{tabular}{lcccccccccc}
\hline & S1 & S2 & S3 & S4 & S5 & S6 & S7 & S8 & S9 & S10 \\
\hline S1 & 1 & 0.85698 & 0.32548 & 0.75421 & 0.26802 & 0.65751 & 0.86591 & 0.54695 & 0.32157 & 0.26985 \\
S2 & 0.85698 & 1 & 0.26587 & 0.41257 & 0.32685 & 0.14573 & 0.86544 & 0.68472 & 0.21965 & 0.32548 \\
S3 & 0.32548 & 0.26587 & 1 & 0.68663 & 0.57862 & 0.6752 & 0.88522 & 0.58562 & 0.87662 & 0.85632 \\
S4 & 0.75421 & 0.41257 & 0.68663 & 1 & 0.85531 & 0.79953 & 0.60218 & 0.86521 & 0.35851 & 0.43651 \\
S5 & 0.21546 & 0.32685 & 0.57862 & 0.85531 & 1 & 0.86201 & 0.6592 & 0.21546 & 0.89751 & 0.77853 \\
S6 & 0.65751 & 0.14573 & 0.6752 & 0.79953 & 0.86201 & 1 & 0.80359 & 0.68845 & 0.25486 & 0.85631 \\
S7 & 0.86591 & 0.86544 & 0.88522 & 0.60218 & 0.6592 & 0.80359 & 1 & 0.88713 & 0.68626 & 0.36681 \\
S8 & 0.54695 & 0.68472 & 0.58562 & 0.86521 & 0.21546 & 0.68845 & 0.88713 & 1 & 0.80031 & 0.8587 \\
S9 & 0.32157 & 0.21965 & 0.87662 & 0.35851 & 0.89751 & 0.25486 & 0.68626 & 0.80031 & 1 & 0.30354 \\
S10 & 0.26985 & 0.32548 & 0.85632 & 0.43651 & 0.77853 & 0.85631 & 0.36681 & 0.8587 & 0.30354 & 1 \\
\hline
\end{tabular}

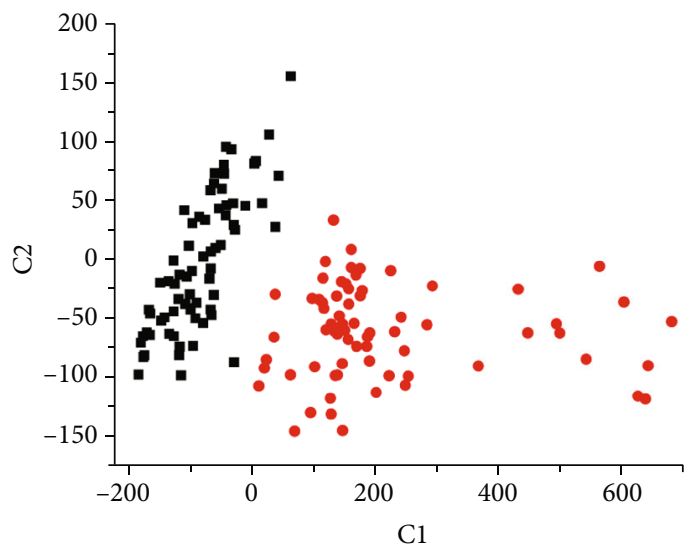

Healthy

Patient

FIGURE 7: PCA for gastritis patients and healthy volunteers.

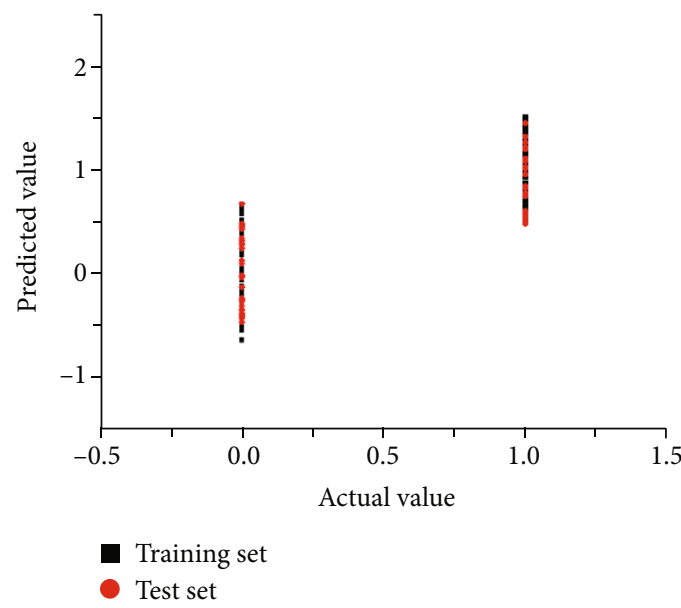

(a) The result of proposed DCNN

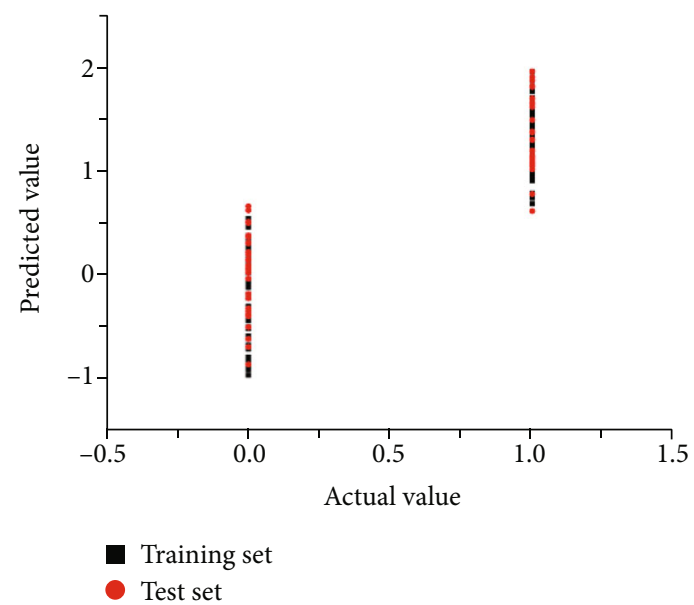

(b) The result of BP

Figure 8: BP and proposed DCNN results for the test sets.

classified, TN is the number of healthy volunteers correctly classified, FN is the number of chronic gastritis misclassified, FP is the number of healthy volunteers misclassified, and $n$ is the total number of samples.
The experiment results are shown in Table 4. As shown in Table 4, it can be seen that the DCNN has nearly the same accuracy compared with BP. But the DCNN has a higher recall rate. This is due to the salient features can be 
TABle 4: Comparison of performances with PCA+BP and DCNN.

\begin{tabular}{lccc}
\hline Model & Accuracy & Sensitivity & Specificity \\
\hline PCA+BP & 0.77 & 0.77 & 0.77 \\
DCNN & 0.85 & 0.90 & 0.80 \\
\hline
\end{tabular}

automatically extracted by the DCNN model, while the success of most other traditional classifiers relies largely on the retrieval of good hand-designed features which is a laborious and time consuming task.

The sensor responses gradually increased during sampling, reaching a peek after $180 \mathrm{~s}$. The BP model only takes the peak values as a characteristic for distinguishing between chronic gastritis patients and healthy volunteers; the sensor's response procedure and sensibility are ignored, which leads to problems later during classification. During the experiment, we found the characteristics of peek values changed due to drifting problem of sensors, which is difficult to obtain good classification results. The proposed DCNN model extracts features from the pattern of sensor responses. The results obtained in the experimental study indicate that e-nose is quite a promising method in the chronic gastritis diagnosis field.

\section{Conclusions}

In this paper, a novel nondestructive diagnostic method for chronic gastritis is proposed by combing e-nose and DCNN. A respiratory gas acquisition system was established to collect respiratory gas samples from patients with gastritis and healthy volunteers. The experimental results show that the proposed method has higher accuracy than the BP method in the detection and identification of chronic gastritis. But the research on the DCNN model to diagnosis chronic gastritis is in its early stage. We hope that this can provide a new thought for a noninvasive detective method for chronic gastritis. The proposed approach can be applied for many sensing application with the help of appropriate sensors. In the future, accuracy and efficiency of the proposed model can be further improved by the fine tuning of its structure and parameters. Some optimizing techniques can also be investigated to boost the classification performance.

\section{Data Availability}

The [sensor data] data used to support the findings of this study have been deposited in the [e-nose] repository (https://github.com/Chenyif/enose).

\section{Conflicts of Interest}

The authors declare that they have no conflicts of interest.

\section{References}

[1] M. M. Ali, N. Hashim, S. A. Aziz, and O. Lasekan, "Principles and recent advances in electronic nose for quality inspection of agricultural and food products," Trends in Food Science \& Technology, vol. 99, pp. 1-10, 2020.
[2] B. D. Hosfield, A. R. Pecoraro, N. T. Baxter, T. B. Hawkins, and T. A. Markel, "The assessment of fecal volatile organic compounds in healthy infants: electronic nose device predicts patient demographics and microbial enterotype," Journal of Surgical Research, vol. 254, pp. 340-347, 2020.

[3] A. Kbmr, B. Lmrg, and A. Lcy, "Application of the electronic nose in predicting preeclampsia in high-risk pregnancies," Pilot study. Archives of Medical Research, vol. 52, no. 5, pp. 561-568, 2021.

[4] P. Maho, C. Herrier, T. Livache, G. Rolland, P. Comon, and S. Barthelmé, "Reliable chiral recognition with an optoelectronic nose," Biosensors \& Bioelectronics, vol. 159, no. 7, article 112183, 2020.

[5] A. Sm, A. Fmg, and B. Bha, "Converting electronic nose into opto-electronic nose by mixing $\mathrm{MoS}_{2}$ quantum dots with organic reagents: application to recognition of aldehydes and ketones and determination of formaldehyde in milk," Analytica Chimica Acta, vol. 1170, no. 25, p. 338654, 2021.

[6] M. Maniscalco and A. Motta, "Clinical and inflammatory phenotyping: can electronic nose and NMR-based metabolomics work at the bedside?," Archives of Medical Research, vol. 49, no. 1, pp. 74-76, 2018.

[7] Y. Sun, M. Zhang, B. Bhandari, and P. Yang, "Intelligent detection of flavor changes in ginger during microwave vacuum drying based on LF-NMR," Food Research International, vol. 119, pp. 417-425, 2019.

[8] J. Troughton and D. Atkinson, "Amorphous InGaZnO and metal oxide semiconductor devices: an overview and current status," Journal of Materials Chemistry C, vol. 7, no. 40, pp. 12388-12414, 2019.

[9] R. M. Nikam, K. H. Kapadnis, and R. Y. Borse, "Tin doped gas sensors in semiconductor metal oxide form and their scientific applications: a review," Journal of Biological Chemistry, vol. 5, no. 3, pp. 53-56, 2019.

[10] T. Zarra, C. Cimatoribus, and V. Naddeo, "Environmental odour monitoring by electronic nose," Global NEST Journal, vol. 20, no. 3, pp. 664-668, 2018.

[11] S. M. Shirsat, G. A. Bodkhe, M. M. Sonawane, B. W. Gawali, and M. D. Shirsat, "Multivariate analysis of a cobalt octaethyl porphyrin-functionalized SWNT microsensor device for selective and simultaneous detection of multiple analytes," Journal of Electronic Materials, vol. 50, no. 10, pp. 57805787, 2021.

[12] M. S. Firouz, K. Mohi-Alden, and M. Omid, "A critical review on intelligent and active packaging in the food industry: research and development," Food Research International, vol. 141, no. 5-6, article 110113, 2021.

[13] J. Fang, G. Yang, and X. Wan, “'Pro-tobacco propaganda': a case study of tobacco industry-sponsored elementary schools in China," Tobacco Control, vol. 29, no. 4, pp. 447-451, 2020.

[14] W. Wei, "Predicting atopic asthma by using eNose breath profiles with machine learning," Journal of Allergy and Clinical Immunology, vol. 146, no. 5, pp. 1010-1012, 2020.

[15] B. Liu, H. Yu, X. Zeng et al., "Lung cancer detection via breath by electronic nose enhanced with a sparse group feature selection approach," Sensors and Actuators B: Chemical, vol. 339, no. 12, article 129896, 2021.

[16] F. Raspagliesi, G. Bogani, S. Benedetti, S. Grassi, S. Ferla, and S. Buratti, "Detection of ovarian cancer through exhaled breath by electronic nose: a prospective study," Cancers, vol. 12, no. 9, p. 2408, 2020. 
[17] W. Li, Z. Jia, D. Xie, K. Chen, J. Cui, and H. Liu, "Recognizing lung cancer using a homemade e-nose: a comprehensive study," Computers in Biology and Medicine, vol. 120, no. 6, article 103706, 2020.

[18] H. Hendrick, R. Hidayat, G.-J. Horng, and Z.-H. Wang, "Noninvasive method for tuberculosis exhaled breath classification using electronic nose," IEEE Sensors Journal, vol. 21, no. 9, pp. 11184-11191, 2021.

[19] J. Abbasi, "Microneedle sensor monitors antibiotics," JAMA The Journal of the American Medical Association, vol. 322, no. 18 , p. $1756,2019$.

[20] R. M. G. E. Goor, J. C. A. Hardy, M. R. A. Hooren, B. Kremer, and K. W. Kross, "Detecting recurrent head and neck cancer using electronic nose technology: a feasibility study," Head \& Neck, vol. 41, no. 9, pp. 2983-2990, 2019.

[21] Y. Xu, J. Jiang, H. Bu et al., "A dual-channel self-calibrating multi-parameter sensor for lung cancer-related exhaled marker rapid identification," Analytical Methods, vol. 12, no. 5, pp. 620-629, 2020.

[22] N. Leunis, M.-L. Boumans, B. Kremer et al., "Application of an electronic nose in the diagnosis of head and neck cancer," Laryngoscope, vol. 124, no. 6, pp. 1377-1381, 2014.

[23] M. D. Zeiler and R. Fergus, Visualizing and Understanding Convolutional Neural Networks, Springer International Publishing, 2014.

[24] P. Moeskops, M. A. Viergever, A. M. Mendrik, L. S. de Vries, M. J. N. L. Benders, and I. Isgum, "Automatic segmentation of MR brain images with a convolutional neural network," IEEE Transactions on Medical Imaging, vol. 35, no. 5, pp. 1252-1261, 2016.

[25] M. Vardhana, N. Arunkumar, S. Lasrado, E. Abdulhay, and G. Ramirez-Gonzalez, "Convolutional neural network for bio-medical image segmentation with hardware acceleration," Cognitive Systems Research, vol. 50, pp. 10-14, 2018.

[26] H. A. Haenssle, J. K. Winkler, C. Fink et al., "Skin lesions of face and scalp - classification by a market-approved convolutional neural network in comparison with 64 dermatologists," European Journal of Cancer, vol. 144, no. 1, pp. 192-199, 2021.

[27] K. Kam, D. M. Rapoport, A. Parekh, I. Ayappa, and A. W. Varga, "WaveSleepNet: an interpretable deep convolutional neural network for the continuous classification of mouse sleep and wake," Journal of Neuroscience Methods, vol. 360, article 109224, 2021.

[28] G. Wei, G. Li, J. Zhao, and A. He, "Development of a LeNet-5 gas identification CNN structure for electronic noses," Sensors, vol. 19, no. 1, p. 217, 2019.

[29] A. Galanty, T. Danel, M. Węgrzyn, I. Podolak, and I. Podolak, "Deep convolutional neural network for preliminary in-field classification of lichen species," Biosystems Engineering, vol. 204, no. 11, pp. 15-25, 2021.

[30] Y. Wang, J. Xing, and S. Qian, "Selectivity enhancement in electronic nose based on an optimized DQN," Sensors, vol. 17, no. 10, p. 2356, 2017.

[31] X. Jiang, P. Jia, R. Luo, B. Deng, S. Duan, and J. Yan, “A novel electronic nose learning technique based on active learning: EQBC- RBFNN," Sensors \& Actuators B Chemical, vol. 249, pp. 533-541, 2017.

[32] Y. Yang, W. Xu, M. Wu, J. Mao, and R. Sha, "Application of Enose combined with ANN modelling for qualitative and quantitative analysis of benzoic acid in cola-type beverages," Jour- nal of Food Measurement and Characterization, vol. 15, no. 6, pp. 5131-5138, 2021.

[33] P. Peng, X. Zhao, X. Pan, and W. Ye, "Gas classification using deep convolutional neural networks," Sensors, vol. 18, no. 2, p. 157, 2018.

[34] L. I. N. Yan, Clinical Study on the Correlation between Halitosis Symptom and Hp of Chronic Superficial Gastritis with Two Pattern of Syndrome, Fujian University Traditional Chinese Medical, Fuzhou, 2009.

[35] X. U. Zhenqin, Detection of Volatile Markers for Non-SmallCell Lung Cancer with Electronic Nose, Anhui Medical University, Anhui, 2009.

[36] L. I. N. Xuejuan, L. I. A. N. G. Lili, and L. I. U. Lisang, "Study on characteristics of odor response patterns between the cold disease nature and heat disease nature of chronic gastritis based on electronic nose," China Jouranl of Traditional Chinese Medicine and Pharmacy, vol. 4, pp. 1193-1197, 2016.

[37] L. I. N. Xuejuan, W. U. Qinghai, and L. I. Candong, "Study on TCM electronic nose based on gas sensors array technology," World Science and Technology/Modernization of Traditional Chinese Medicine and Materia Medica, vol. 5, pp. 1992-1995, 2012.

[38] W. Hu, Y. Huang, L. Wei, F. Zhang, and H. Li, "Deep convolutional neural networks for hyperspectral image classification," Journal of Sensors, vol. 2015, 2015.

[39] Y. Lu, S. Yi, N. Zeng, Y. Liu, and Y. Zhang, "Identification of rice diseases using deep convolutional neural networks," Neurocomputing, vol. 267, pp. 378-384, 2017.

[40] S. Ioffe and C. Szegedy, Batch Normalization: Accelerating Deep Network Training by Reducing Internal Covariate Shift, pp. 448-456, 2015.

[41] I. Sutskever, J. Martens, and G. Dahl, "On the importance of initialization and momentum in deep learning," in International Conference on International Conference on Machine Learning, 2013http://JMLR.org.

[42] A. Daffertshofer, C. J. C. Lamoth, O. G. Meijer, and P. J. Beek, "PCA in studying coordination and variability: a tutorial," Clinical biomechanics, vol. 19, no. 4, pp. 415-428, 2004.

[43] X. Xie and K. M. Lam, "Gabor-based kernel PCA with doubly nonlinear mapping for face recognition with a single face image," IEEE Transactions on Image Processing, vol. 15, no. 9, pp. 2481-2492, 2006. 